\title{
Observation of coupled plasmon- polariton modes of plasmon waveguides for electromagnetic energy transport below the diffraction limit
}

Stefan A. Maier, Pieter G. Kik, Harry A. Atwater, Sheffer Meltzer, Aristides A. G. Requicha, et al.

Stefan A. Maier, Pieter G. Kik, Harry A. Atwater, Sheffer Meltzer, Aristides A. G. Requicha, Bruce E. Koel, "Observation of coupled plasmon-polariton modes of plasmon waveguides for electromagnetic energy transport below the diffraction limit," Proc. SPIE 4810, Properties of Metal Nanostructures, (4 October 2002); doi: 10.1117/12.450799

SPIE Event: International Symposium on Optical Science and Technology, 2002, Seattle, WA, United States 


\title{
Observation of coupled plasmon-polariton modes of plasmon waveguides for electromagnetic energy transport below the diffraction limit
}

\author{
Stefan A. Maier*a ${ }^{\text {a }}$ Pieter G. Kik ${ }^{\mathrm{a}}$, Harry A. Atwater ${ }^{\mathrm{a}}$, \\ Sheffer Meltzer ${ }^{b}$, Ari A.G. Requicha ${ }^{b}$, Bruce E. Koel ${ }^{b}$ \\ ${ }^{a}$ Thomas Watson Laboratory of Applied Physics, California Institute of Technology, Pasadena, CA \\ ${ }^{\mathrm{b}}$ Laboratory for Molecular Robotics, University of Southern California, Los Angeles, CA
}

\begin{abstract}
We investigate the possibility of using arrays of closely spaced metal nanoparticles as plasmon waveguides for electromagnetic energy below the diffraction limit of light. Far-field spectroscopy on arrays of closely spaced $50 \mathrm{~nm} \mathrm{Au}$ particles fabricated using electron beam lithography reveals the presence of near-field optical particle interactions that lead to shifts in the plasmon resonance frequencies for longitudinal and transverse excitations. We link this observation to a point-dipole model for energy transfer in plasmon waveguides and give an estimate of the expected group velocities and energy decay lengths for the fabricated structures. A near-field optical excitation and detection scheme for energy transport is proposed and demonstrated. The fabricated structures show a high propagation loss of about $3 \mathrm{~dB} / 15 \mathrm{~nm}$ which renders a direct experimental observation of energy transfer impossible. The nature of the loss and ways to decrease it by an order of magnitude are discussed. We also present finite-difference time-domain simulations on the energy transfer properties of plasmon waveguides.
\end{abstract}

Keywords: plasmon waveguides, metal nanoparticles, near-field optics, NSOM, surface plasmon, plasmon-polariton

\section{INTRODUCTION}

The miniaturization of optical devices to size dimensions akin to their electronic counterparts is a major goal of current research efforts in optoelectronics, photonics and semiconductor manufacturing. High integration of optical components for the fabrication of all-optical chips requires both a confinement of the guided optical modes to small dimensions and the ability to route energy around sharp corners. Confinement and guiding of electromagnetic energy (EM) at optical frequencies has been achieved in spatial dimensions in the micron and sub-micron regime using both planar waveguide technology ${ }^{1}$ and photonic crystals. ${ }^{2}$ Whereas planar waveguides do not allow for the guiding of light around sharp corners with a bending radius much smaller than the wavelength $\lambda$ of the guided mode, bend engineering of defect modes in photonics crystals has enabled the fabrication of complex guiding geometries. ${ }^{2}$ The size and density of optical devices employing these technologies is nonetheless limited by the diffraction limit $\lambda / 2 n$ of light, which imposes a lower size limit of a few hundred nanometers on the optical mode size.

Recently, a new method for the guiding of EM energy that allows for optical mode dimensions below the diffraction limit has been proposed, based on near-field interactions between closely spaced noble metal nanoparticles (spacing $d<<$ $\lambda$ ) that can be efficiently excited at their surface plasmon frequency. ${ }^{3}$ The guiding principle relies on coupled plasmonpolariton modes set up by near-field dipole interactions that lead to coherent propagation of energy along the array. An analytical point-dipole model allowed for the characterization of the guiding properties of such "plasmon waveguides" in terms of the dispersion relation (frequency $\omega$ versus wavevector $\mathbf{k}$ ) of the guided modes, and group velocities for energy transport of about $10 \%$ of the velocity of light and energy decay lengths on the order of $3 \mathrm{~dB} / 500 \mathrm{~nm}$ have been predicted for linear arrays of Ag particles in air. ${ }^{4}$ Plasmon waveguides could allow for guiding of EM energy in a variety of sub-wavelength structures such as corners and tee-structures, and an all-optical switch based on interference between coherent plasmon-polariton waves has been proposed. ${ }^{4,5} \mathrm{We}$ verified the possibility of guiding EM energy using periodic 
metal structures with $d<<\lambda$ in a macroscopic analogue operating in the microwave regime and demonstrated the feasibility of the proposed corner and tee guiding geometries. ${ }^{6}$ An analysis of the far-field spectroscopic properties of arrays consisting of Au nanoparticles with a diameter $D=50 \mathrm{~nm}$ and a center-to-center spacing $d=75 \mathrm{~nm}$ fabricated using electron beam lithography allowed us to estimate the maximum group velocity for energy transport to about $4 \mathrm{x}$ $10^{6} \mathrm{~m} / \mathrm{s}$, corresponding to an energy decay length of $3 \mathrm{~dB} / 15 \mathrm{~nm} .{ }^{7}$ For an application of plasmon waveguides in nanoscale optical circuits, a propagation loss decrease of at least one order of magnitude is desirable in order to allow for energy guiding over several hundred nanometers. We will show in a forthcoming publication that this can be achieved via a careful geometry change of the nanoparticles constituting the waveguides.

In this article, we report on our efforts on the investigation of the guiding properties of plasmon waveguides. Section 2 gives a concise review of the theory of the plasmon excitation of noble metal nanoparticles with a special emphasis on a solid understanding of the loss processes. We describe a characterization method of the guiding properties of plasmon waveguides based on far-field polarization spectroscopy and near-field optical microscopy together with finitedifference time-domain (FDTD) simulations in section 3. We end by summarizing the present status of our work on plasmon waveguides.

\section{THE SURFACE PLASMON RESONANCE}

\subsection{Position of the dipole surface plasmon resonance:}

It is well established that single noble metal nanoparticles interact strongly with visible light when resonantly excited at their surface plasmon frequency. ${ }^{8}$ The resonant behavior is due to the confinement of the conduction electrons inside the particle which sets up an effective restoring force due to surface polarization upon a light-induced displacement of the conduction electrons. For small particles ( $D<15 \mathrm{~nm}$ for Ag and $D<25 \mathrm{~nm}$ for Au particles) retardation of the driving light field along the particle volume is negligible and all conduction electrons are excited inphase in a dipole-like oscillation. Quasistatic Mie-theory allows for the calculation of the far-field extinction cross section $\sigma_{\mathrm{ext}}$ for a spherical metal nanoparticle of volume $V_{0}$ and dielectric function $\varepsilon(\omega)=\varepsilon_{1}(\omega)+\mathrm{i} \varepsilon_{2}(\omega)$ embedded in a non-absorbing medium with dielectric constant $\varepsilon_{\mathrm{m}}$ :

$$
\sigma_{\mathrm{ext}}=9 \frac{\omega}{c} \varepsilon_{m}^{\frac{3}{2}} V_{0} \frac{\varepsilon_{2}(\omega)}{\left[\varepsilon_{1}(\omega)+2 \varepsilon_{m}\right]^{2}+\varepsilon_{2}(\omega)^{2}}
$$

For small damping $\left(\varepsilon_{2}(\omega), \frac{\partial \varepsilon_{2}}{\partial \omega}<<1,\right)$ the resonance occurs when the Fröhlich condition

$$
\varepsilon_{1}(\omega)=-2 \varepsilon_{m}
$$

is met. For small damping, the resonance position is only dependent on the real part $\varepsilon_{1}$ of the metal dielectric function and can be shifted throughout the visible and near-infrared range by embedding the particle in hosts with different dielectric constants $\varepsilon_{\mathrm{m}}$. For non-spherical particles in the dipole limit $(D<<\lambda)$, the resonance condition (2) is modified to account for the change in boundary conditions at the particle surface. For spheroids, this results in short-axes and longaxes dipole plasmon modes which are shifted with respect to the plasmon dipole resonance frequency of a spherical particle (Figure 1). Let us note that whereas for free electron metals the dipole resonance frequency $\omega_{0}$ depends only on the real part $\varepsilon_{1}(\omega)$ of the dielectric function and the surrounding medium $\varepsilon_{\mathrm{m}}$, and the resonance width only on $\varepsilon_{2}(\omega)$, for realistic metals both resonance position and width in general depend on $\varepsilon_{1}$ and $\varepsilon_{2}$. 


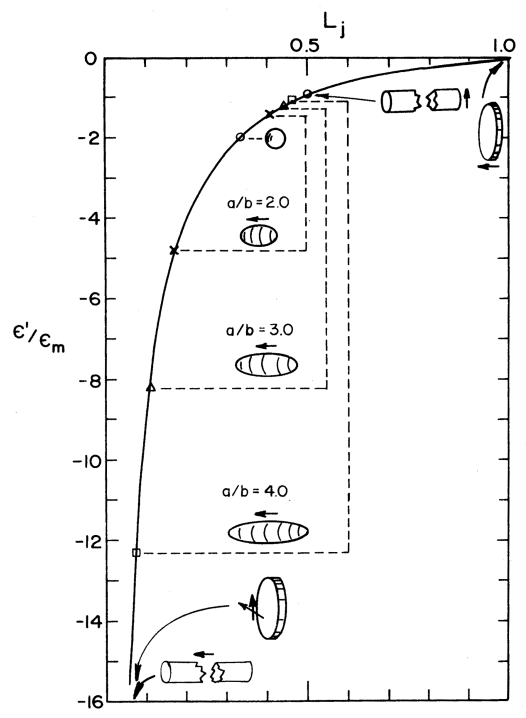

Figure 1. Dipole resonance position for spheroids with different aspect ratios 1:1, 2:1, 3:1 and 4:1 for both the long-and the short-axis mode of excitation. After ref. 9.

\subsection{Plasmon damping:}

The damping of a surface plasmon resonance of a metal nanoparticle is due to the dephasing of the coherent conduction electron motion with time. Figure 2 shows a schematic of the different processes involved in the damping of a surface plasmon excitation excited by a photon of energy $\mathrm{h} v$.

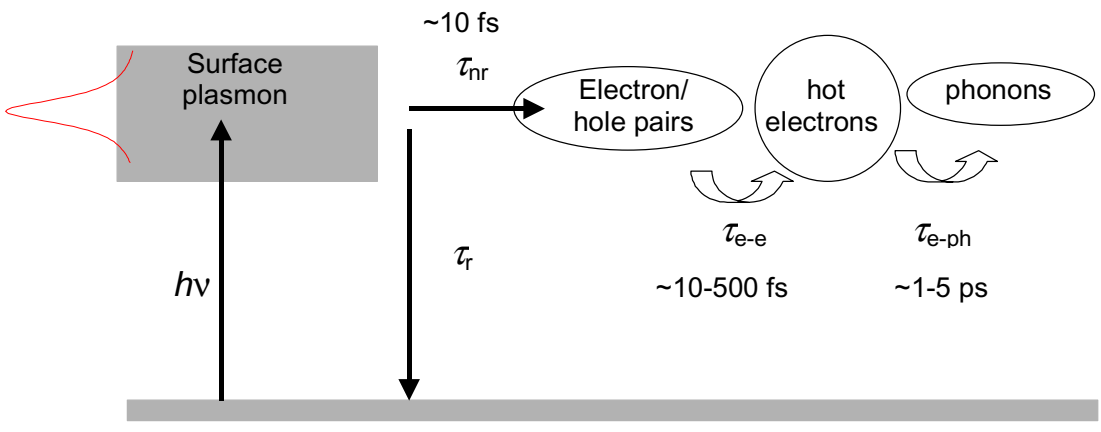

Figure 2. Energy relaxation of a surface plasmon. The relaxation takes place either via radiative or non-radiative processes. The nonradiative relaxation channel leads to the creation of electron-hole pairs and the subsequent relaxation into hot electrons and phonons.

The damping of the plasmon resonance is in general described via a total dephasing time $T_{2}$ and an energy relaxation time $T_{1}{ }^{10}$ Both are related to the homogeneous linewidth $\Gamma$ of the surface plasmon resonance via

$$
\Gamma(\omega)=\frac{2}{T_{2}}=\frac{1}{T_{1}}+\frac{1}{T_{2}^{*}} .
$$


$T_{2}{ }^{*}$ is called the "pure dephasing time" which describes quasi-elastic electron scattering events that change the electron wavevector but not its energy. Its contribution to the total dephasing time $T_{2}$ is often put into the energy relaxation time $T_{1}$, yielding $T_{2}=2 T_{1}$. The damping of the plasmon resonance is thus determined by the energy relaxation time $T_{1}$, defined as

$$
\frac{1}{T_{1}}=\frac{1}{\tau_{n r}}+\frac{1}{\tau_{r}}
$$

The energy relaxation of a plasmon oscillation is composed of a non-radiative decay channel with timeconstant $\tau_{\text {nr }}$ and a radiative decay channel with timeconstant $\tau_{\mathrm{r}}$. For metal nanoparticles with a diameter $\mathrm{D}<<\lambda$ the energy relaxation is mostly due to non-radiative processes $\left(\tau_{\mathrm{r}}>>\tau_{\mathrm{nr}}\right){ }^{10}$ The oscillating electrons loose their energy via inelastic scattering to single electron excitations (Landau damping), so called electron-holes states with a timeconstant $\tau_{\mathrm{nr}}$ on the order of 10 fs. These transitions can either be intraband or interband, and increase with increasing spectral overlap of the plasmon resonance with the interband absorption edge. The electron-hole states subsequently relax via electron-electron and electron-phonon scattering on the $100 \mathrm{fs}$ and 1 ps timescale.

The plasmon damping of noble metal nanoparticles with diameters $5 \mathrm{~nm}<D<30 \mathrm{~nm}$ is mostly due to non-radiative processes and can thus be expressed via the materials parameters such as the optical conductivity or the dielectric function. Kreibig derived the following analytical approximation for the linewidth $\Gamma(\omega)$ based on the optical conductivity as the only damping mechanism: ${ }^{11}$

$$
\Gamma(\omega)=\frac{2 \varepsilon_{2}(\omega)}{\sqrt{\left(\frac{\partial \varepsilon_{1}}{\partial \omega}\right)^{2}+\left(\frac{\partial \varepsilon_{2}}{\partial \omega}\right)^{2}}}(1+\eta)
$$

where usually $\eta<<1$ holds. For free-electron metals described via a Drude model, $\Gamma$ reduces to the Drude relaxation frequency $\gamma$. Figure 3 shows the plasmon decay times $\mathrm{T}_{1}$ calculated with (5) for $\mathrm{Au}(\mathrm{a})$ and $\mathrm{Ag}$ (b) nanoclusters using literature values of the dielectric functions. ${ }^{12-14}$
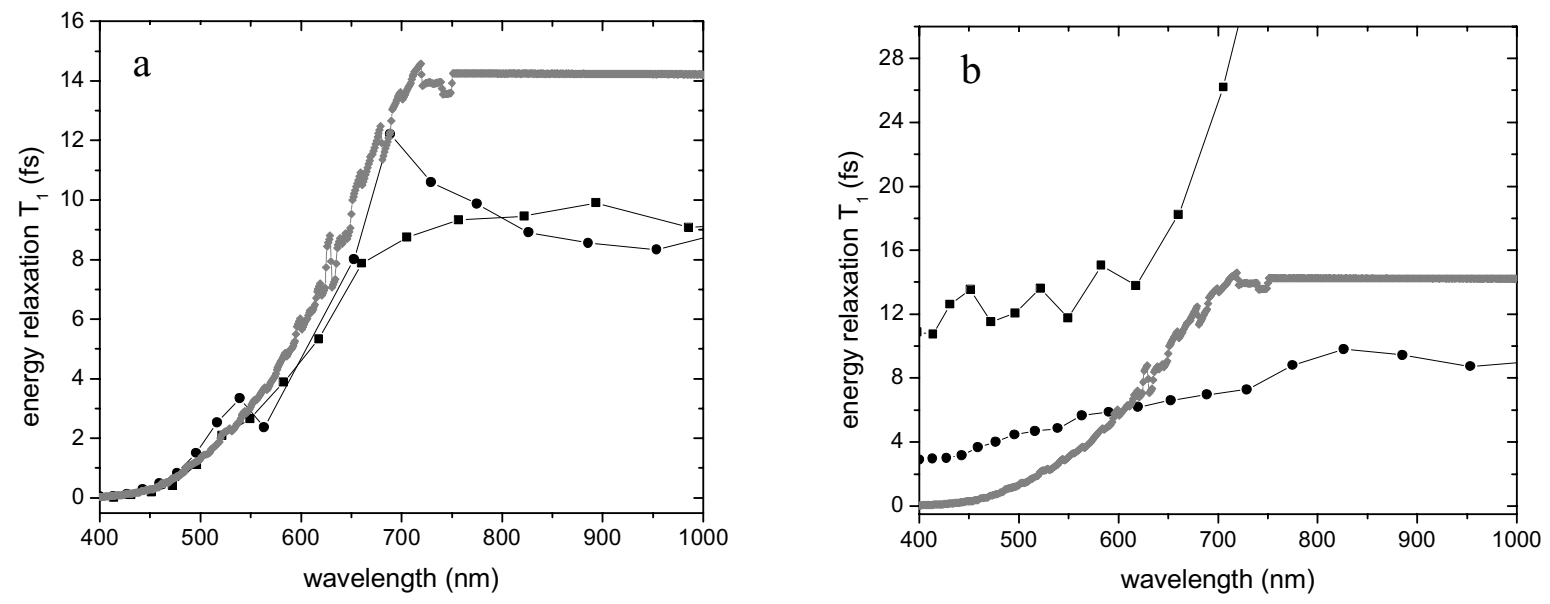

Figure 3. Plasmon decay time for $\mathrm{Au}(\mathrm{a})$ and $\mathrm{Ag}$ (b) nanoparticles calculated via (5) using dielectric functions of Ref. 12 (black squares), 13 (black circles) and 14 (grey diamonds)

For Au nanoparticles (Figure 3a), there is a close agreement between the decay times calculated using the different sets of literature values for the dielectric function for wavelengths $\lambda<700 \mathrm{~nm}$. The strong damping of the plasmon resonance 
below $700 \mathrm{~nm}$ is due to the absorption tail of the Au interband transitions which extends from the bandedge at $2.5 \mathrm{eV}$ $(\sim 500 \mathrm{~nm})$ down to $1.7 \mathrm{eV}(725 \mathrm{~nm})$. In this regime, a competition between plasmon excitation and d-sp interband absorption prevents efficient plasmon oscillations. Indeed, plasmon decay times of $4 \mathrm{fs}$ have been reported for $\mathrm{Au}$ nanoparticles with a resonance wavelength $\lambda_{0}=632 \mathrm{~nm}$ on a $\mathrm{TiO}_{2}$ matrix. ${ }^{15}$ For Ag nanoparticles (Figure $3 \mathrm{~b}$ ), there is a marked difference between the decay times calculated using the bulk dielectric data by reference 12 and references 1314 throughout the regime of optical wavelengths. Measurements of the decay time of silver clusters with resonance wavelengths $400 \mathrm{~nm}<\lambda_{0}<630 \mathrm{~nm}$ yielded decay times between 2 and $4 \mathrm{fs},{ }^{16}$ suggesting the use of the dielectric functions of references 13-14 for estimations of the plasmon damping. Let us again note that the decay times reported here are based solely on the optical conductivity as a damping mechanism (scattering of the electrons) and give thus only an upper limit for the plasmon decay due to dephasing and interband transitions. Further decay processes such as Landau-damping and radiation damping lead to a shortage of the plasmon decay time $T_{1}$. Radiation damping leading to energy relaxation via the emission of photons is of increasing importance for larger particles $(D>50 \mathrm{~nm})$ and leads to a red - shift and a broadening of the dipole resonance. For very small particles $(D<5 \mathrm{~nm})$, mostly elastic surface scattering events lead to a significant resonance broadening due to a decrease of the pure dephasing time. For very small clusters on substrates, chemical interface damping can be the dominating decay channel. ${ }^{17}$

In summary, plasmon decay times $T_{1}<10 \mathrm{fs}$ can be expected for $\mathrm{Au}$ and $\mathrm{Ag}$ nanoparticles throughout the visible regime. This will limit the maximum size of waveguides based on plasmon-polariton excitations in noble metal nanoparticles to the submicron regime. ${ }^{3,4}$

\section{FABRICATION AND CHARACTERIZATION OF PLASMON WAVEGUIDES}

\subsection{Energy transfer in plasmon waveguides:}

The dipole surface plasmon resonance of a single noble metal nanoparticle can be employed for a field localization and enhancement in the vicinity of the resonantly excited nanoparticle. For small nanoparticles $(D<<\lambda)$ the metal nanoparticle acts as an oscillating dipole. The field enhancement at the particle surface is proportional to the decay time $T_{1}$ of the surface plasmon oscillation and is confined to the near-field region of the particle over distances much smaller than the wavelength of light. If a second metal nanoparticle is placed in this near field region, the interaction strength between the particles is greatly enhanced. Indeed, it has been observed that a chain of closely spaced Au nanoparticles shows a collective behavior when excited in total internal reflection geometry, with an enhanced near-field between adjacent particles. ${ }^{18}$ Figure 4 shows a finite-difference time-domain (FDTD) simulation of the distribution of the $\mathrm{x}$ component the electric field around a linear array of 5 Au nanoparticles with a diameter $D=50 \mathrm{~nm}$ and a center-tocenter spacing $d=75 \mathrm{~nm}$ in air. The particles are excited in-phase at their collective plasmon resonance frequency with a plane wave propagating along the $\mathrm{z}$-direction with the electric field polarized in the $\mathrm{x}$-direction (along the axis of the array). Details about the simulation technique and the determination of the collective plasmon resonance frequencies of nanoparticle arrays can be found elsewhere. ${ }^{19}$

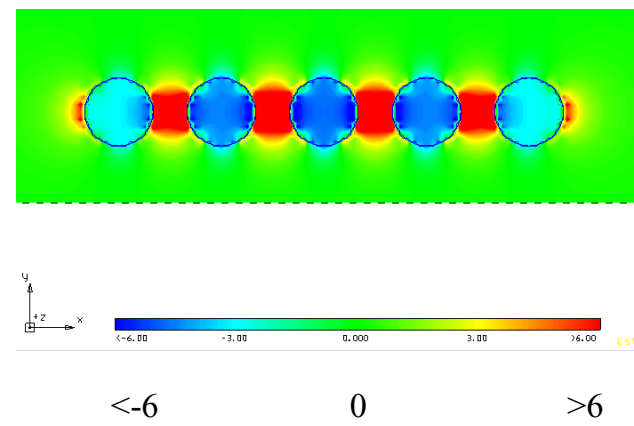

Figure 4. Distribution of the $\mathrm{x}$-component of the electric field around an array of $5 \mathrm{Au}$ nanoparticles in air excited at the collective plasmon resonance frequency by a propagating plane wave with the electric field polarized in the x-direction. The field enhancement inside and between the particles is due to dipolar near-field interactions. 
The collective dipole resonance frequency of the array differs from the resonance frequency $\omega_{0}$ of a single nanoparticle due to near-field interactions between the closely spaced particles, ${ }^{7,19}$ and a field enhancement on the order of 10 between the metal nanoparticles is observed.

We fabricated arrays of closely spaced Au nanoparticles $(D=50 \mathrm{~nm})$ with variable center-to-center spacings $d$ in order to investigate the distance-dependence of the near-field coupling and to validate our simulation results. The fabrication method of choice was electron-beam-lithography on ITO-coated glass, providing a good control over particle shape and inter-particle spacing. ${ }^{5}$ Figure 5a shows a scanning electron micrograph of particle arrays consisting of 80 nanoparticles with a spacing $d=75 \mathrm{~nm}$. The particles are almost spherical in shape, resulting in a negligible optical anisotropy. ${ }^{7}$

a)

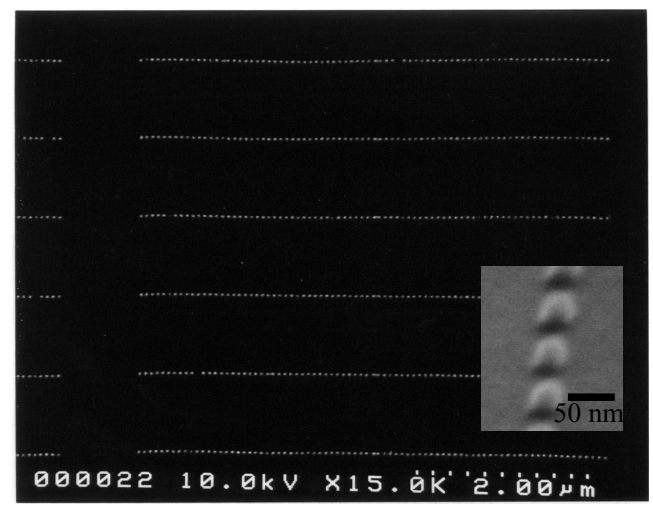

b)

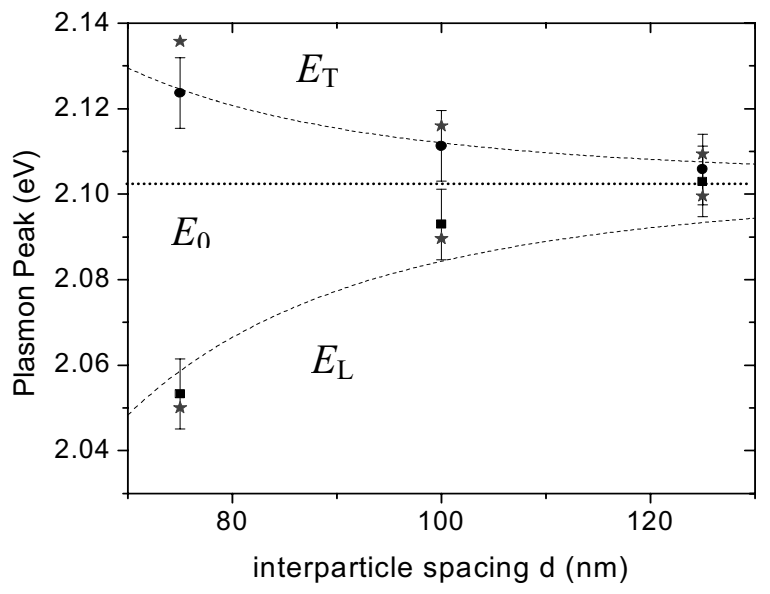

Figure 5. a) Scanning electron micrograph of Au nanoparticle arrays fabricated using electron beam lithography on ITO coated glass. The individual particles are $50 \mathrm{~nm}$ in diameter and spaced $75 \mathrm{~nm}$ apart. The inset shows a micrograph of individual particles taken under a 70 degree angle. b) Collective plasmon resonance energies for both longitudinal ( $E_{\mathrm{L}}$, black squares) and transverse $\left(E_{\mathrm{T}}\right.$, black circles) excitations of Au nanoparticle arrays of different spacings $d$ obtained via far-field spectroscopy and finite-difference timedomain simulations (grey stars). The collective resonances are shifted with respect to the single particle resonance energy $E_{0}$ and follow a $d^{-3}$ dependence (dotted line).

Figure $5 \mathrm{~b}$ shows the energies $E_{\mathrm{L}, \mathrm{T}}$ of the collective dipole surface plasmon resonances for nanoparticle arrays with interparticle spacings $d$ of 75, 100 and $125 \mathrm{~nm}$ for a polarization of the electric field along (longitudinal modes L) and perpendicular (transverse modes T) to the array axis obtained via far-field spectroscopy ${ }^{7}$ and FDTD simulations (grey stars). The longitudinal resonances $E_{\mathrm{L}}$ (black squares) are shifted to lower and the transverse resonances $E_{\mathrm{T}}$ (black circles) to higher energies with respect to the single particle resonance energy $E_{0}$ due to near-field optical interactions between the particles constituting the arrays. ${ }^{7}$ The peak shifts $\Delta E_{\mathrm{L}, \mathrm{T}}=\left|E_{0}-E_{\mathrm{L}, \mathrm{T}}\right|$ decrease with increasing interparticle spacing as $d^{-3}$ and are a direct measurement of the particle interaction strength.

The measured collective plasmon resonance energies $E_{\mathrm{L}, \mathrm{T}}$ can be related to the dispersion relation for energy transport in plasmon waveguides using a point-dipole model. ${ }^{4}$ This dipole-model treats the interactions between adjacent nanoparticles as point-dipolar interactions with a $d^{-3}$ dependence and allows for the determination of the dispersion relation (frequency $\omega$ versus wavevector $\mathbf{k}$ ) for propagating plasmon waves along the nanoparticle arrays. Figure 6 shows this dispersion relation for both the longitudinal mode $\mathrm{L}$ and the transverse mode $\mathrm{T}$ taking into account interactions between nearest-neighbors only (solid curves) and interactions between 5 nearest neighbors (dotted line). As expected, nearest-neighbor interactions dominate the dispersion properties in the point-dipole model. 


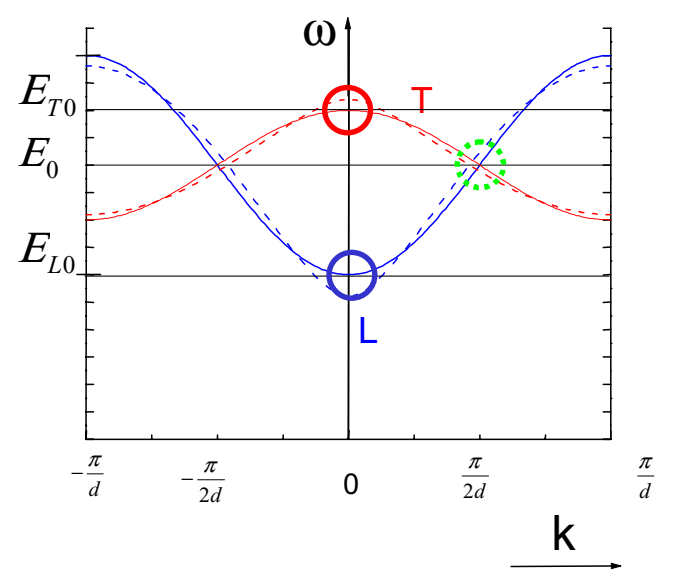

Figure 6. Dispersion relation for energy transport in plasmon waveguides for both the longitudinal mode $\mathrm{L}$ and the transverse mode $\mathrm{T}$ calculated using a point-dipole model with nearest-neighbor interactions (solid lines) and interactions between 5 nearest neighbors (dotted lines). ${ }^{4}$ The highlighted points at $\mathbf{k}=0$ (solid circles) can be probed using far-field spectroscopy, and the $\mathbf{k}=\pi / 2 d$ modes (dotted circle) are the modes of highest group velocity for energy transport.

Since all the particles are excited in phase in the far-field illumination geometry, the energies $E_{\mathrm{L}, \mathrm{T}}$ of the collective plasmon resonances obtained using far-field spectroscopy can be related to the $\mathbf{k}=0$ long-wavelength modes of the dispersion relation (highlighted by solid circles in Figure 6). The peak shifts $\Delta E_{\mathrm{L}, \mathrm{T}}$ are thus a direct measurement of the bandwidths $B_{\mathrm{L}, \mathrm{T}}=2 \times \Delta E_{\mathrm{L}, \mathrm{T}}$ available for energy transport.

The dispersion relation allows for a determination of the group velocity $v_{\mathrm{g}}$ (given by the slope $\partial \omega / \partial \mathbf{k}$ ) and the plasmon wave attenuation length $\alpha$ for energy transport in plasmon waveguides. For in-phase excitation at $\mathbf{k}=0$, the group velocity is zero and no energy transport takes place, prohibiting a direct measurement of $v_{\mathrm{g}}$ using far-field spectroscopy. The determined bandwidth $B_{\mathrm{L}, \mathrm{T}}$ however allows for a determination of the maximum group velocity $v_{\mathrm{maxL}, \mathrm{T}}$ at $\mathbf{k}=\pi / 2 d$ using the point-dipole model. ${ }^{4}$ It can be shown that for $B_{\mathrm{L}, \mathrm{T}}$ expressed in $\mathrm{rad} / \mathrm{s}$

$$
v_{\max L, T}=\frac{B_{L, T} \times d}{2}
$$

The attenuation length $\alpha$ of the propagating plasmon waves depends on the homogeneous linewidth $\Gamma$ of the plasmon oscillation and the group velocity $v_{\mathrm{g}}$. For the mode of maximum group velocity it can be shown that ${ }^{20}$

$$
\alpha_{L, T} \times d=\frac{\Gamma}{B_{L, T}}
$$

Both the maximum group velocity $v_{\max }$ and the attenuation length $\alpha$ for energy transport can thus be expressed using the quantities $B$ and $\Gamma$ that can be determined using spectroscopy. Whereas far-field spectroscopy allows for a direct determination of the bandwidth $B$ as described above, the homogeneous linewidth $\Gamma$ can only be probed via far-field spectroscopy if no inhomogeneous broadening due to a size dispersion of the nanoparticles constituting the plasmon waveguides is present. The samples fabricated using electron beam lithography generally fulfill this condition, allowing for a direct determination of $\Gamma$ from a far-field extinction measurement. ${ }^{7}$ We verified the linewidth obtained from such a far-field measurement via fluorescence near-field spectroscopy as depicted in Figure 7. 


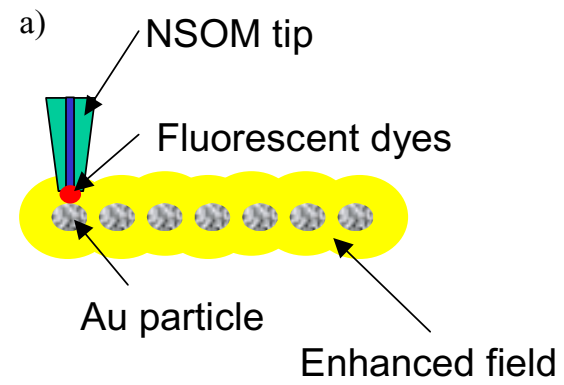

b)

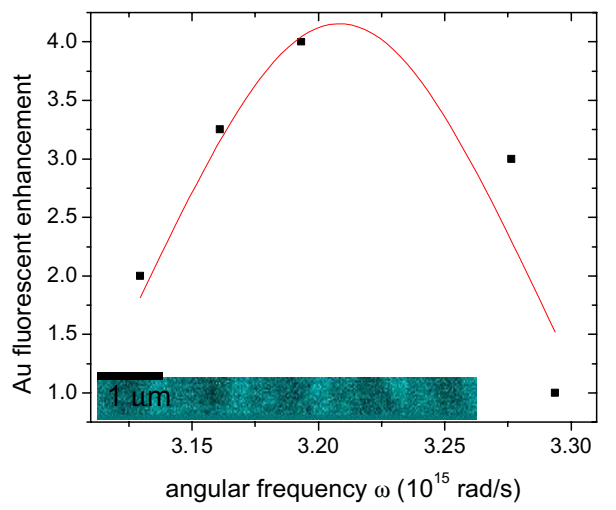

Figure 7. Fluorescence near-field spectroscopy of plasmon waveguides. a) The Au nanoparticles are excited with the tip of a scanning near-field optical microscope (NSOM) in the vicinity of their plasmon resonance. The enhanced field around the particles is probed via fluorescent beads attached to the NSOM tip. b) Fluorescence intensity versus excitation frequency of the laser light, showing the homogenous lineshape of the the plasmon resonance. The inset shows the fluorescent intensity variation in a scan over 4 plasmon waveguides spaced 1 micron apart.

Figure 7 a shows a schematic of our setup. We use the tip of a near-field optical microscope ${ }^{21}$ as a local excitation source to probe the properties of single nanoparticles constituting the plasmon waveguides. An excitation laser frequency in the vicinity of the single particle dipole resonance leads to the build-up of an enhanced electromagnetic field around the particle structure. This local field is probed via latex beads attached to the tip filled with fluorescent molecules that show a constant absorption in the vicinity of the dipole resonance frequency. The emitted intensity of fluorescent radiation is thus a direct measurement of the local field. ${ }^{22}$ Figure $7 \mathrm{~b}$ shows the enhancement of the fluorescent intensity as the excitation frequency is scanned through the dipole resonance frequency of the Au particles. The obtained intensity data can be fitted to a Lorentz - lineshape with a linewidth of about $180 \mathrm{meV}$, corresponding to a decay time of $3.6 \mathrm{fs}$, which is in close agreement to measurements of the homogeneous linewidth for Au nanoparticles on a TiO $\mathrm{Substrate}^{15}$

Using the described spectroscopic measurements, we determined a maximum group velocity of $4 \mathrm{x} 10^{6} \mathrm{~m} / \mathrm{s}$ and a energy decay length of $3 \mathrm{~dB} / 15 \mathrm{~nm}$ for the longitudinal $\mathbf{k}=\pi / 2 d$ mode for the fabricated plasmon waveguides consisting of $50 \mathrm{~nm}$ Au particles with a interparticle spacing $d=75 \mathrm{~nm}$. $^{7}{ }^{20}$ The current particle geometry can thus not produce long range energy transfer for the selected materials. Ways to substantially decrease this loss using spheroidal Au particles that show a longer plasmon decay time and an increased interparticle coupling are discussed elsewhere. ${ }^{20}$ Instead, in the remaining part of this paper we want to focus on a local detection scheme for energy transport using both experiments and FDTD simulations.

\subsection{Local excitation of plasmon waveguides:}

In order to directly examine the transport properties of plasmon waveguides, a local excitation of a non-zero wavevector $\mathbf{k}$ is necessary as opposed to an in-phase excitation of the particle arrays as a whole at $\mathbf{k}=0$ using far-field illumination as described above. To accomplish this we locally excite a nanoparticle in the fabricated nanoparticle arrays using the tip of an illumination mode NSOM. Figure 8a shows a schematic of our excitation and detection setup. Power transport away from the excited nanoparticles is probed via the careful placement of polystyrene nanobeads filled with fluorescent molecules in close proximity to the nanoparticle structures using the tip of an atomic force microscope. ${ }^{23}$ The fluorescent dyes absorb at $590 \mathrm{~nm}$ near the plasmon resonance frequency of a single fabricated Au particle and emit radiation at $610 \mathrm{~nm}$, which is detected in the far-field. This scheme allows the observation of energy transport properties of plasmon waveguides since energy gets transferred from the illuminating tip to the plasmon waveguide, gets transferred along the nanoparticle structure and finally excites a fluorescent bead placed at a sufficient distance from the excitation source. Details about the experimental setup can be found elsewhere. ${ }^{23}$ 


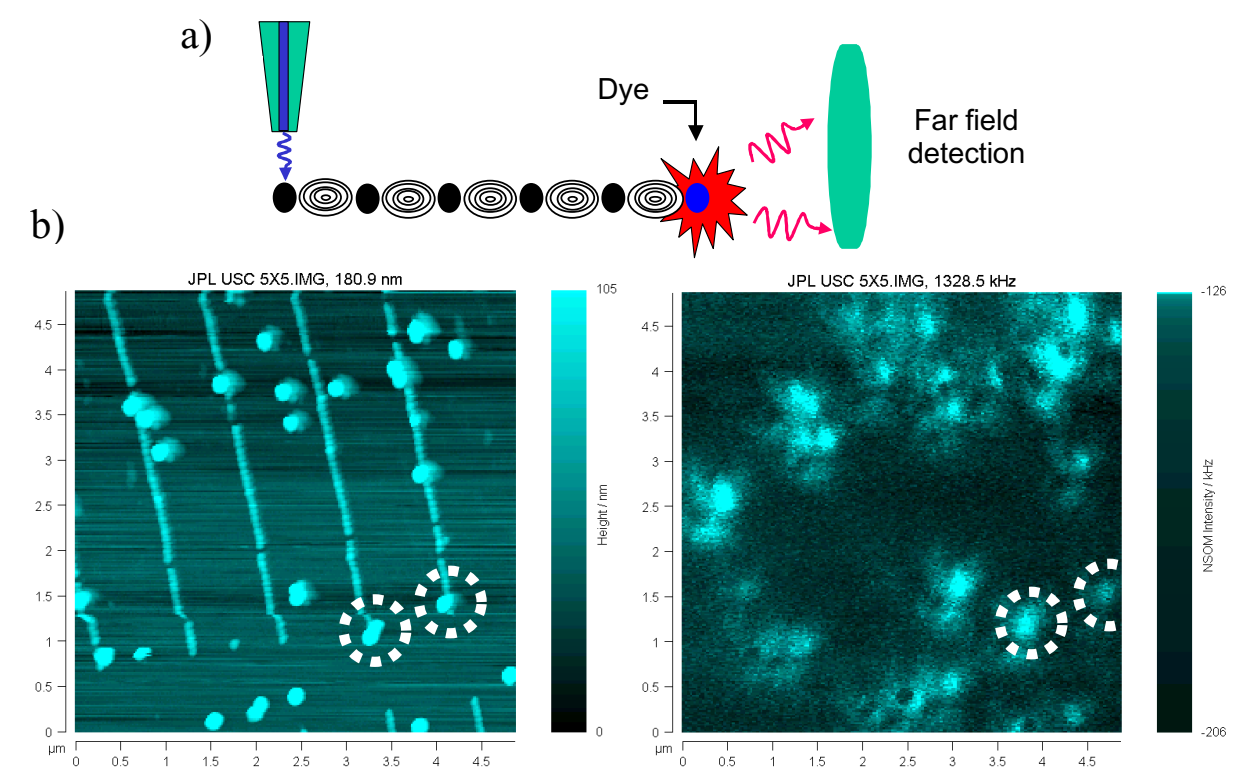

Figure 8. a) Schematic of the near-field excitation and far-field fluorescence detection scheme. b) topographic (left) and fluorescence intensity (right) plot of a $5 \times 5$ micron scan of fabricated plasmon waveguides with randomly deposited $100 \mathrm{~nm}$ dye beads. Two beads were pushed to the end of the nanoparticle arrays (white dotted circles) using the tip of an atomic force microscope.

Figure $8 \mathrm{~b}$ shows the topography (left) the detected and fluorescent intensity (right) of a 5 x 5 micron scan of the fabricated plasmon waveguide samples consisting of $50 \mathrm{~nm}$ Au particles with a spacing $d=75 \mathrm{~nm}$. Single Au nanoparticles can be resolved in the topography image, which also shows the randomly deposited $100 \mathrm{~nm}$ polystyrene fluorescent particles. Two particles were pushed to the end of two plasmon waveguides (indicated with white circles) using the tip of an atomic force microscope. The fluorescent image shows single particle dye bead luminescence if the tip is positioned directly over the dye particles. However, the dynamic range of the fluorescent far-field detection is not big enough to observe signs of energy transport along the nanoparticles constituting the plasmon waveguides due to the high energy loss estimated to $3 \mathrm{~dB} / 15 \mathrm{~nm}$, corresponding to an energy loss of $97 \%$ between adjacent nanoparticles. For spheroidal nanoparticles, a decreased energy loss of about $3 \mathrm{~dB} / 130 \mathrm{~nm}$ is expected, ${ }^{20}$ which could possibly be detected using the proposed detection scheme. We are currently working on the fabrication of corresponding plasmon waveguides with spheroidal particle geometries.

The transport properties of plasmon waveguides can also be numerically examined in FDTD simulations as described above by using a local dipole excitation source placed in close proximity to the nanoparticle arrays instead of plane-wave illumination. This way, all modes of the dispersion relation are accessible. Figure 9 shows an initial example of a waveguide consisting of $950 \mathrm{~nm}$ Au particles with a center-to-center spacing $d=75 \mathrm{~nm}$ in air. A point-dipole polarized in the x-direction (longitudinal mode) and driven at the resonance frequency $\omega_{0}$ of a single Au nanoparticle is placed $75 \mathrm{~nm}$ to the left of the nanoparticle array, providing thus a continuous local excitation source. Figure 9 shows the $\mathrm{x}$-component of the electric field around the plasmon waveguide structure $60 \mathrm{fs}$ after starting the continuous excitation. A mode profile with $\mathbf{k}=\pi / 2 d$ is clearly visible along the nanoparticle structure, in agreement with the dispersion relation calculated using the analytical point-dipole model discussed above. 


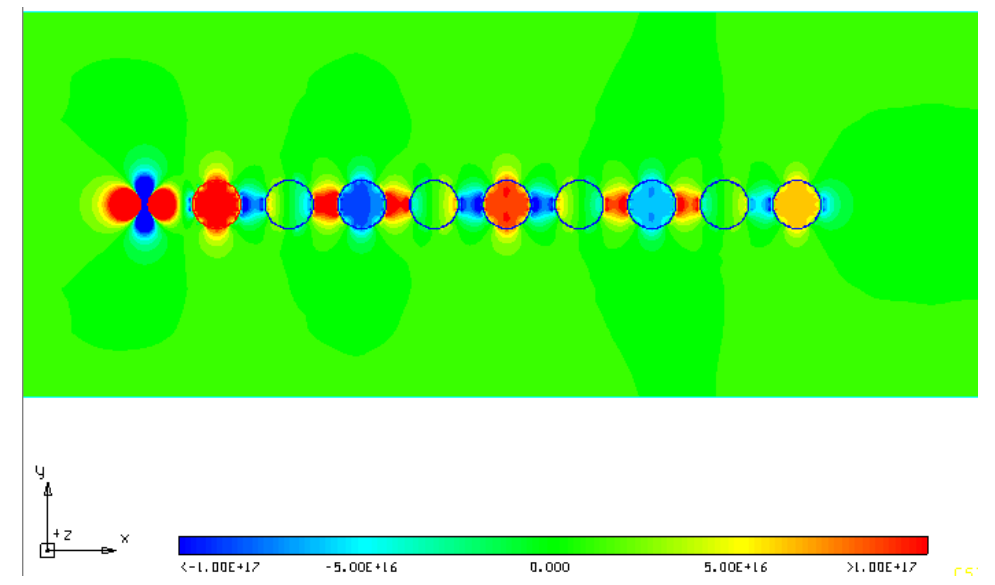

Figure 9. Finite-difference time-domain simulation of a plasmon waveguide consisting of $950 \mathrm{~nm}$ Au particles spaced $75 \mathrm{~nm}$ apart. The particles are locally excited by a point dipole with longitudinal polarization placed $75 \mathrm{~nm}$ to the left of the nanoparticle array. The dipole frequency is set equal to the plasmon resonance frequency of a single nanoparticle, thus exciting the plasmon waveguide in the $\mathbf{k}=\pi / 2 d$ mode in agreement with the predictions from the point-dipole dispersion relation.

We are currently working on the determination of the full dispersion relation of plasmon waveguides using FDTD simulation with both continuous and pulsed local excitation. The results will be published shortly in a forthcoming article.

\section{CONCLUSIONS}

We have investigated the possibility of using arrays of closely spaced noble metal nanoparticles as plasmon waveguides for electromagnetic energy transfer below the diffraction limit of light. Arrays of closely spaced Au nanoparticles fabricated using electron beam lithography exhibit shifts in their collective plasmon resonance frequencies due to near-field particle interactions, and a theory connecting the spectroscopic data with the expected energy guiding parameters has been presented. Whereas the interparticle interaction is not strong enough to allow direct experimental observation of energy transfer using near-field optical microscopy, finite-difference time-domain simulations allow for a careful examination of the energy localization and guiding properties of plasmon waveguides.

\section{ACKNOWLEDGEMENTS}

We would like to thank Richard Muller and Paul Maker of the Jet Propulsion Laboratory in Pasadena for expert technical assistance. This work was sponsored by the NSF grant ECS0103543 and the Center for Science and Engineering of Materials at Caltech and the Air Force Office of Scientific Research.

\section{REFERENCES}

1. B.E.A. Saleh and M.C. Teich, The Fundamentals of Photonics, Wiley, New York, 1991

2. Attila Mekis, J.C. Chen, I. Kurland, Shanhui Fan, Pierre R. Villeneuve, and J.D. Joannopoulos, "High transmission through sharp bends in photonics crystal waveguides", Physical Review Letters 77, pp 3787-3790, 1996

3. M. Quinten, A. Leitner, J.R. Krenn, and F.R. Aussenegg, "Electromagnetic energy transport via linear chains of silver nanoparticles", Optics Letters 23, pp 1331-1333, 1998

4. M.L. Brongersma, J.W. Hartman, and H.A. Atwater, "Electromagnetic energy transfer and switching in nanoparticle chain arrays below the diffraction limit", Physical Review B 62, R16356-R16359, 2000 
5. S.A. Maier, M.L. Brongersma, P.G. Kik, S. Meltzer, A.A.G. Requicha, and H.A. Atwater, "Plasmonics - a route to nanoscale optical devices", Advanced Materials 13, pp 1501-1505, 2001

6. S.A. Maier, M.L. Brongersma, and H.A. Atwater, "Electromagnetic energy transport along arrays of closely spaced metal rods as an analogue to plasmonic devices", Applied Physics Letters 78, pp 16-18 (2001)

7. S.A. Maier, M.L. Brongersma, P.G. Kik, and H.A. Atwater, "Observation of near-field coupling in metal nanoparticle chains using far-field polarization spectroscopy”, Physical Review B 65, 193408 (2002)

8. U. Kreibig and M. Vollmer, Optical Properties of Metal Clusters, Springer, New York, 1994

9. C. Bohren and D. Huffman, Absorption and Scattering of Light by Small Particles, Wiley \& Sons, New York (1998)

10. E.J. Heilweil and R.M. Hochstrasser, "Nonlinear spectroscopy and picosecond transient grating study of colloidal gold”, Journal of Chemical Physics 82, pp 4762-4770, 1985

11. U. Kreibig, "Small silver particles in photosensitive glass: their nucleation and growth", Applied Physics 10, pp 255-264, 1976

12. P.B. Johnson and R.W. Christy, "Optical constants of the noble metals", Physical Review B 6, pp 4370-4379, 1972

13. E.D. Palik, Handbook of Optical Constants, Academic Press, London, 1985

14. M. Quinten, "Optical constants of gold and silver clusters in the spectral range between $1.5 \mathrm{eV}$ and $4.5 \mathrm{eV}$, Zeitschrift für Physik B 101, pp 211-217 (1996)

15. T. Klar, M. Perner, S. Grosse, G. von Plessen, W. Spirkl, and J. Feldmann, "Surface-plasmon resonances in single metallic nanoparticles", Physical Review Letters 80, pp 4249-4252 (1998)

16. T.J. Silva, Master's thesis, UCSD, 1994

17. H. Hövel, S. Fritz, A. Hilger, U. Kreibig, and M. Vollmer, "Width of cluster plasmon resonances: bulk dielectric functions and chemical interface damping”, Physical Review B 48, 18178-18188, 1993

18. J.R. Krenn, A. Dereux, J.C. Weeber, E. Bourillot, Y. Lacroute, J.P. Goudonnet, G. Schider, W. Gotschy, A. Leitner, F.R. Aussenegg, and C. Girard, "Squeezing the optical near-field zone by plasmon coupling of metallic nanoparticles”, Physical Review Letters 82, 2590-2593, 1999

19. S.A. Maier, P.G. Kik, M.L. Brongersma, H.A. Atwater, S. Meltzer, A.A.G. Requicha, and B.E. Koel, “Observation of coupled plasmon-polariton modes of plasmon waveguides for electromagnetic energy transport below the diffraction limit", Materials Research Society Symposium Proceedings 722, L6.2.1, 2002

20. S.A. Maier, P.G. Kik, and H.A. Atwater, "Observation of coupled plasmon-polariton modes in Au nanoparticle chain waveguides of different lengths: Estimation of waveguide loss", Applied Physics Letters (submitted)

21. K. Lieberman, N. Ben-Ami, and A. Lewis, "A fully integrated near-field optical, far-field optical, and normal-force scanned probe microscope”, Review of Scientific Instruments 67, pp 3567-3572, 1996

22. P. Das and A. Puri, "Energy flow and fluorescence near a small metal particle", Physical Review B 65, 115416 , 2002

23. S.A. Maier, P.G. Kik, M.L. Brongersma, and H.A. Atwater, "Electromagnetic energy transport below the diffraction limit in periodic metal nanostructures”, Proceedings of SPIE 4456, 22-30, 2001

*e-mail: stmaier@caltech.edu 